\title{
Digitale Strukturen im sozio-technischen Experimentierfeld
}

\author{
Perspektiven der Technischen Universität Hamburg
}

Das nutzerinnen- und nutzerorientierte sozio-technische Experimentierfeld der Technischen Universität Hamburg (TU Hamburg) konnte nur im Kontext der Hamburg Open Online University (HOOU) entwickelt werden. Um das sozio-technische Experimentierfeld zu verstehen, braucht es daher ein Verständnis über dessen kontextuelle Verortung - sowohl innerhalb wie auch außerhalb der Organisation.

\section{Ermöglichungsstrukturen für das sozio-technische Experimentierfeld der HOOU an der TU Hamburg}

Wenngleich oder weil Hochschulen zunehmend im Wettbewerb miteinander stehen, sei es in der Exzellenzinitiative, in der Akquise von Drittmitteln oder um (internationale) Studierende, kooperieren sie auch zunehmend in verschiedenen Handlungsfeldern. Seit den 1990er Jahren - im Übrigen dieselbe Zeit, in der das New Public Management eingeführt wurde - wurden vermehrt themenund bundeslandbezogene Netzwerke, Hochschulverbünde und Landesinitiativen gegründet. Dabei fallen aktuell insbesondere Netzwerke und Kooperationen auf, an denen (fast) alle Hochschulen eines Bundeslandes beteiligt sind, um das Thema Digitalisierung gemeinsam zu gestalten.

Im Rahmen des Forschungsprojektes BRIDGING ${ }^{1}$ konnten in elf Bundesländern aktuelle Hochschulinitiativen identifiziert werden, die sich dem Thema Digitalisierung in Studium und Lehre widmen. Neun davon sind formalisierte Netzwerke, die von fast allen öffentlich-rechtlichen Hochschulen in Landesträgerschaft durch unterschiedlichste Arbeitsstrukturen gemeinsam gestaltet werden, allerdings unterschiedlichste Handlungsfelder und Selbstverständnisse aufweisen. $^{2}$ Darüber hinaus finden sich in zwölf Bundesländern Hochschuldidaktikzentren beziehungsweise Lehrverbünde (siehe Tabelle 1).

1 https://bridging.rz.tuhh.de/.

2 Brandenburg hat ein nicht formalisiertes Netzwerk der E-Learning-Beauftragten von sechs Hochschulen. Die hochschulübergreifenden Qualifizierungen in Thüringen werden durch die

ə Open Access. (C) 2020 Tina Ladwig und Christiane Arndt, published by De Gruyter. @) BY-NC-ND This work is licensed under the Creative Commons Attribution-NonCommercial-NoDerivatives 4.0 License. 
Tab. 1: Überblick zu identifizierten Landesinitiativen (Stand: Dezember 2019).

\begin{tabular}{ll}
\hline Bundesland & Hochschulverbund zu Digitalisierung \\
\hline Baden-Württemberg & Hochschulnetzwerk Digitalisierung der Lehre (HND BW) \\
Bayern & Virtuelle Hochschule Bayern (vhb) \\
Berlin & nein \\
Brandenburg & E-Learning Netzwerk Brandenburg [eBB] \\
Bremen & nein \\
Hamburg & Hamburg Open Online University (HOOU)/ \\
& Multimedia Kontor Hamburg (MMKH) \\
Hessen & Digital gestütztes Lehren und Lernen in Hessen \\
Mecklenburg-Vorpommern & nein \\
Niedersachsen & E-Learning Academic Network Niedersachsen (ELAN e. V.) \\
Nordrhein-Westfalen & Digitale Hochschule NRW (DH.NRW) \\
Rheinland-Pfalz & Virtueller Campus Rheinland-Pfalz (vcrp) \\
Saarland & nein \\
Sachsen & Bildungsportal Sachsen/ Arbeitskreis E-Learning \\
Sachsen-Anhalt & Netzwerk digitale Hochschullehre im Rahmen von Heteroge- \\
& nität als Qualitätsherausforderung für Studium und Lehre \\
& (HET LSA) \\
Schleswig-Holstein & nein \\
Thüringen & eteach-Netzwerk zur Qualifizierung im Bereich digitale Hoch- \\
& schuldidaktik \\
\hline
\end{tabular}

An einem dieser Hochschulverbünde - der Hamburg Open Online University ist auch die TU Hamburg beteiligt. Die HOOU versteht sich als „Netzwerk“3 ${ }^{\text {“3 }}$ von mittlerweile fünf der sechs staatlichen Hamburger Hochschulen in Landesträgerschaft, der Behörde für Wissenschaft, Forschung und Gleichstellung (BWFG), der Senatskanzlei sowie dem Multimedia Kontor Hamburg (MMKH). ${ }^{4}$

Während die Impulse zur kooperativen Auseinandersetzung mit dem Thema Digitalisierung in der Lehre in Hamburg vor allem angesichts des zunehmenden Einflusses privatwirtschaftlicher Unternehmen aus der Landespolitik gekommen sind, kann die inhaltliche Ausrichtung des Verbundes auf die Aushandlungsprozesse zwischen Vertreterinnen beziehungsweise Vertretern der Landespolitik, des MMKH und der Hochschulen, die der Gründung der HOOU vorausgingen, zurückgeführt werden.

Universität Weimar entwickelt und angeboten. IT-Netzwerke wurden (noch) nicht in die Recherche einbezogen.

3 www.hoou.de. Die folgende Darstellung basiert auf den Informationen, die auf der Webseite der HOOU zur Verfügung gestellt werden.

4 Die Universität Hamburg ist seit April 2019 nicht mehr Teil der HOOU. 
Dabei wurde erkannt, dass die Hochschulen mit denselben Fragen und Herausforderungen durch Digitalisierung konfrontiert sind und eine einzelne Hochschule diese nicht alleine bewältigen kann. Zum anderen bestand - ausgehend vom MOOC-Hype - der Wunsch der Hochschulen, nicht einfach Formate zu imitieren, sondern etwas Eigenes zu entwickeln. Dieser Aushandlungsprozess führte 2014 zu einem Konzept für die HOOU.

Die HOOU nahm, wie in der Strategie „Digitale Stadt“ ${ }^{\star 5}$ beschlossen, ihre Arbeit 2015 auf. Von 2015 bis Ende 2016 wurde sie als Vorprojekt gefördert, um mit allen Akteurinnen und Akteuren das gemeinsame Konzept weiterzuentwickeln und die technische Basis zu schaffen. Ziel war es, Synergien zu nutzen und gegebenenfalls fehlende Strukturen zu etablieren. In diesem Rahmen wurden die ersten „Early Bird Projekte“ durch die HOOU gefördert. ${ }^{6} 2017$ bis 2018 wurde die HOOU als Projekt weitergeführt. 2019 wurde die Landesinitiative offiziell verstetigt.

Die HOOU geht der Frage nach, wie man in Zukunft lernt. Die digitalen Lernangebote sollen:

- interdisziplinäre und hochschulübergreifende Bearbeitung von Aufgaben, Problemstellungen und Projekten ermöglichen,

- klassische Präsenzlehre ergänzen,

- frei zugänglich sein und

- einen digitalen Raum für Studierende, Lehrende und alle Interessierten schaffen.

Den „Markenkern“ der HOOU bilden dabei vier Leitideen:

1. Lernendenorientierung und Kollaboration

Die Erstellung der Lernangebote erfolgt ausgehend von den Merkmalen und Zielen der Lernenden und spezifischen Problemstellungen und Themenfeldern.

2. Wissenschaftlichkeit

Die Lernangebote werden auf akademischem Niveau erstellt und fördern das wissenschaftliche Denken und Arbeiten.

5 Das ressortübergreifende Landesstrategiepapier „Die Digitalisierung der großen Stadt Chancen für Wirtschaftskraft, Kommunikation und öffentliche Dienstleistungen“ wurde 2015 von der Senatskanzlei auf Deutsch und Englisch veröffentlicht. Es werden Felder und Projekte benannt, in denen Hamburg bereits aktiv ist und weiterhin aktiv sein wird. https://www.hamburg.de/contentblob/9260384/ed1cb41d024dbef3f62bd9cd834ca838/data/strategie-deutsch. pdf.

6 Vgl. hierzu den Sonderband der HOOU-Content-Projekte: http://epub.sub.uni-hamburg.de/ epub/volltexte/2018/76395/pdf/hoou_content_projekte_2015_20161.pdf. 
3. Öffnung für neue Zielgruppen und zivilgesellschaftliche Relevanz Die Lernangebote ermöglichen die Auseinandersetzung mit akademischen Inhalten für alle Interessierten - unabhängig von einer Hochschulzugehörigkeit.

4. Openness/OER

Alle Lernangebote werden als Offene Bildungsressourcen (Open Educational Resources (OER)) angeboten, die weiterverwendet und -entwickelt werden können. Zudem sollen vor allem Open-Source-Software und offene Lizenzen genutzt werden.

\section{Umsetzungsstrukturen des sozio-technischen Experimentierfeldes der $\mathrm{HOOU}$ an der TU Hamburg}

\section{Institutionelle Verankerung}

Die HOOU steht für das Ausprobieren neuer Konzepte und Formate digitalen Lehrens und Lernens. Wenngleich alle entwickelten Lernangebote auf der zentralen Plattform ${ }^{7}$ abgebildet werden, können Hochschulen unter Berücksichtigung des Markenkerns selbst entscheiden, mit welchen Umsetzungsansätzen sie die Leitideen inhaltlich und technisch gestalten.

Den Hochschulen wurde hierbei viel Freiraum gewährt, Offenheit, Transparenz, zivilgesellschaftliche Relevanz, OER und Kollaboration in ihrem Kontext zu definieren. Somit entstanden an allen Hochschulen erste Ideen und Konzepte $\mathrm{zu}$ webgestützten Lehr- und Lernangeboten. Diese dezentralen Strukturen sind zum einen darauf zurückzuführen, dass sich die HOOU-Plattform noch im Aufbau und in Planung befand und zum anderen, dass ein solcher Raum für innovative Lehr- und Lernangebote nur sukzessive und immer in Referenz zu den Bedarfen der Lehrenden und Lernenden erschlossen werden kann.

So ist ein Ziel der HOOU, aus den prototypischen Entwicklungen und Umsetzungen offener, digital gestützter Lehr- und Lernangebote Anforderungen an eine hochschularten- und fachdisziplinübergreifende Plattform abzuleiten und die Diversität der Angebote der HOOU für Lernende aber auch Lehrende zu fördern. Während die Hochschule für Angewandte Wissenschaften Hamburg sich beispielsweise insbesondere auf Qualifizierung, Kompetenzorientierung und

7 www.hoou.de. 
OER fokussiert, konzentriert sich die TU Hamburg vor allem auf die Umsetzung eines offenen sozio-technischen Experimentierfelds. Das Experimentierfeld kann dabei als Raum in der Präsenz und im Digitalen verstanden werden, in dem offene Lehr- und Lernangebote entwickelt und betreut werden. Im Rahmen sozialer Aushandlungsprozesse mit Lehrenden und Lernenden, aber auch mediendidaktischen Beraterinnen und Beratern werden neue Formen digital gestützter Lehr-Lernprozesse konzeptionell entwickelt. Diese Aushandlungsprozesse wiederum sind die Basis von Bedarfen, die sich dann wiederum auch in technischen Strukturen abbilden. Dreh- und Angelpunkt der technischen Strukturen im Experimentierfeld ist, dass diese auch die ausgehandelten Werte von Offenheit, Partizipation und Kollaboration widerspiegeln und ermöglichen. Aus technischer Perspektive entstehen in den Experimentierfeldern somit digitale Strukturen, die aus der losen Kopplungen bedarfsorientiert eingesetzter Tools entstehen. Je nach Lehr- und Lernangebot können sich diese Strukturen auch immer wieder verändern, erweitert oder auch verdichtet werden.

Mittlerweile sind die Experimentierfelder an den Hochschulen wesentlicher Bestandteil der Umsetzungsstrukturen der HOOU. Demnach besteht die HOOU aus zwei Handlungsfeldern, die parallel zueinander laufen und gleichzeitig didaktisch-konzeptionell und technisch miteinander abgestimmt werden (Schwalbe et al. 2016).

\section{Portal der HOOU}

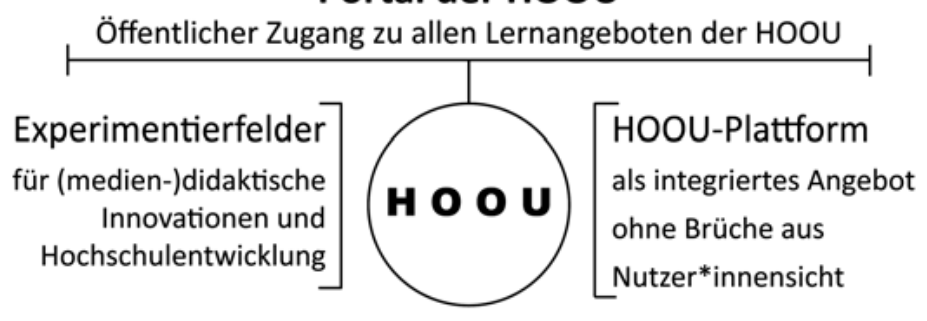

Abb. 1: Konzeptionelle Verortung der Experimentierfelder.

In den sozio-technischen Experimentierfeldern ist somit das Ziel (medien-)didaktische Innovation zu fördern. Essentiell hierfür sind - neben den finanziellen Ressourcen - engagierte Akteurinnen und Akteure in den Hochschulen, das heißt zentrale HOOU-Teams oder HOOU-Angehörige, die in den Hochschulen Beratungs- und Umsetzungsunterstützung leisten für die Lehrenden aus den verschiedenen Fachdisziplinen, die im Sinne des Markenkerns der HOOU in ihren Projekten innovative Formen medienbasierten Lehrens und Lernens an den Hochschulen vorantreiben und offene Lehr- und Lernangebote entwickeln und 
erproben $^{8}$. Durch die geschaffenen Freiräume, sich mit dem Markenkern der HOOU hochschul- und fachspezifisch auseinanderzusetzen, kann sich auch die Umsetzung einzelner HOOU-Projekte stark voneinander unterscheiden. Ziel dieses explorativen Vorgehens ist die Förderung der Innovationskraft.

Ein solches exploratives Vorgehen bedarf auch entsprechender Methoden bei der Konzeption, Entwicklung und Implementierung der Lehr-Lernsettings. Die TU Hamburg greift hierbei einen partizipatorischen Ansatz auf, der aus der agilen Softwareentwicklung stammt: dem sogenannten User Story Mapping (USM) (Patton 2014). Dieses Vorgehen ermöglicht es, frühzeitig ein möglichst umfassendes Bild von den Vorstellungen der unterschiedlichen Nutzenden hinsichtlich Online-Werkzeugen und Mediennutzung zu erhalten und die Akzeptanz der Angebote bei den späteren Lernenden früh zu ermitteln (Dürkop/Ladwig 2016a; Dürkop/Ladwig 2016b). Dies bedeutet, dass zunächst die relevanten Stakeholder, die an dem Lernangebot beteiligt sind, identifiziert werden. Hierzu zählen neben den Lehrenden aus den jeweiligen Fachdisziplinen, die das Projekt initiiert haben, auch die Lernenden. Im Fall der HOOU bedeutet dies, dass nicht nur die Studierenden der jeweiligen Hochschulen zu berücksichtigen sind, sondern auch die Zielgruppen, die außerhalb der Hochschulen an den Lehr- und Lernangeboten teilnehmen können. Im Fall des HOOU-Projektes Knifellix ${ }^{9}$ sind das Schülerinnen und Schüler, deren Eltern, aber auch Pädagoginnen und Pädagogen. Die Lernenden werden explizit in den Prozess der Materialerstellung einbezogen und lernen somit nicht nur, sich ihren eigenen Lernprozess zu strukturieren, sondern auch essentielle Digitalkompetenzen, wie zum Beispiel kollaborative Arbeitsweisen, das Analysieren und Reflektieren von und über Inhalte, aber auch problem- und vielmehr auch lösungsorientiertes Handeln.

Da die Entwicklung von Lernangeboten, die dem HOOU-Markenkern entsprechen, noch weitere Anforderungen mit sich bringen, sind auch Juristinnen und Juristen, Vertreterinnen und Vertreter der Rechenzentren, der Bibliotheken oder auch der Öffentlichkeitsarbeit mit einzubeziehen. So heterogen die Rollen, so heterogen sind auch die Vorkenntnisse dieser Personen hinsichtlich der Nutzung digitaler Medien. Somit gilt es nun im Rahmen eines USM-Workshops die unterschiedlichen Vorstellungen einzufangen und zu einer gemeinsamen Geschichte zusammenzuführen. Der Workshop selbst ist in drei Phasen unterteilt und verfolgt das Schema, dass alle ihre individuellen Vorstellungen formulieren, die dann in der Gruppe diskutiert werden, so dass sich ein geteiltes Verständnis für das Lernarrangement herausbildet (Ladwig/Dürkop 2016a).

8 http://hoou.tuhh.de.

9 https://kniffelix.rz.tu-harburg.de. 
Einen Einblick in einen solchen Workshop an der Hochschule für bildende Künste (HFBK) zeigt Abbildung 2.

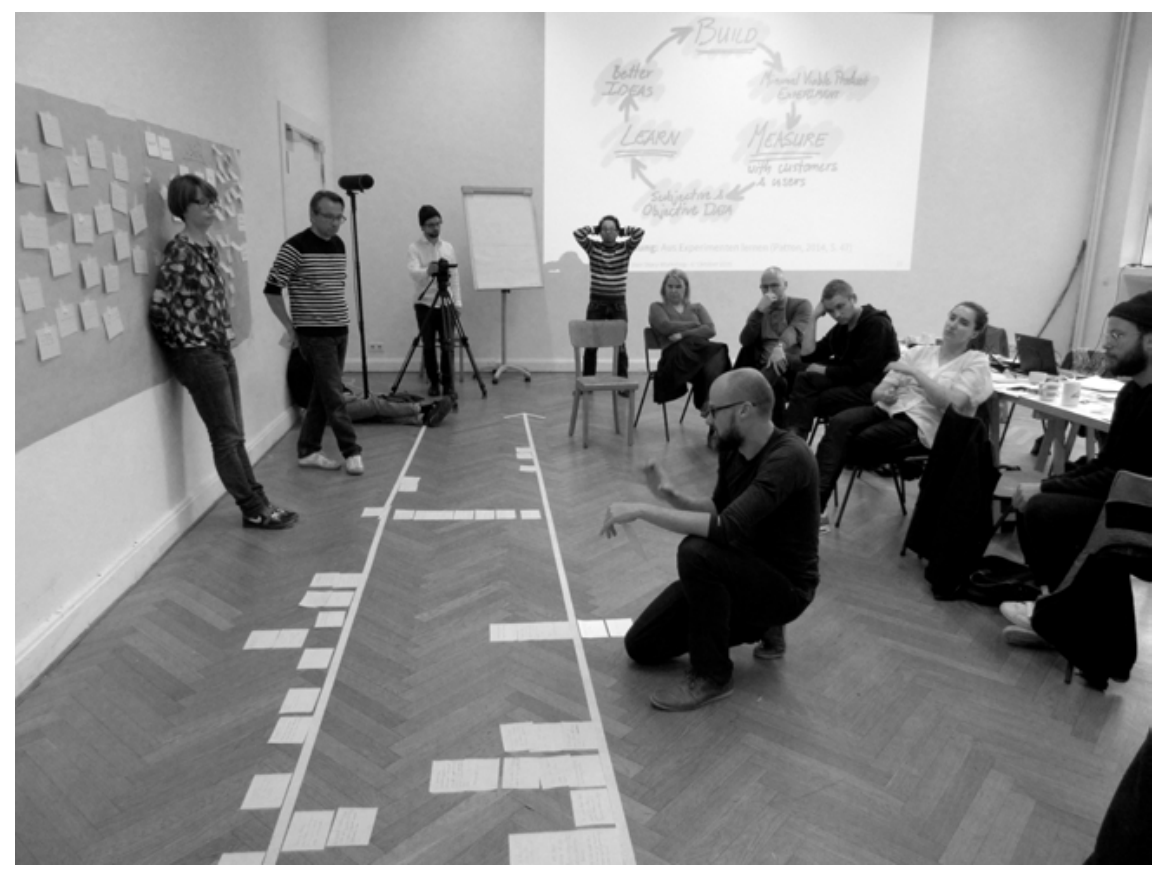

Abb. 2: Momentaufnahme aus einem USM-Workshop an der Hochschule für bildende Künste (HFBK).

An der TU Hamburg sind im Rahmen der entwickelten Lernprojekte für die HOOU innovative Formate entstanden, die besonders zum Austausch und damit zu einem Kulturwandel in der TU Hamburg beigetragen haben. Die Leitidee, Freiräume zum Experimentieren zu schaffen, ist dabei auch technisch hinterlegt. So wurde zusammen mit der TU-Bibliothek und dem TU-Rechenzentrum ein Experimentierfeld konzipiert, welches nunmehr von unterschiedlichen Instituten genutzt wird, um neue Lehr- und Lernformate auszuprobieren. Technisch beruht dieses Experimentierfeld auf der Kombination verschiedener Open Source Tools. Diese Kombination basiert zum einen auf den Prämissen des Markenkerns der HOOU und zum anderen auf den individuellen fachspezifischen Anforderungen der HOOU-Projekte. Im Zentrum steht die weltöffentliche GitLab-Instanz ${ }^{10}$. Ziel hierbei war und ist es, die Quellen von Open Educational Re-

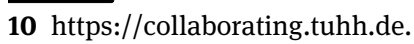


sources (OER), Forschungsdaten und weiteren digitalen Artefakten unkompliziert und weltweit zugänglich zu machen. Über diesen Zugang wiederum können hochschul- und fachdisziplinübergreifende Kooperationen im nationalen wie auch internationalen Kontext angestoßen werden. Neben GitLab werden aber auch offene Foren ${ }^{11}$ oder virtuelle Simulationsumgebungen ${ }^{12}$ angeboten (Dürkop/Ladwig 2018).

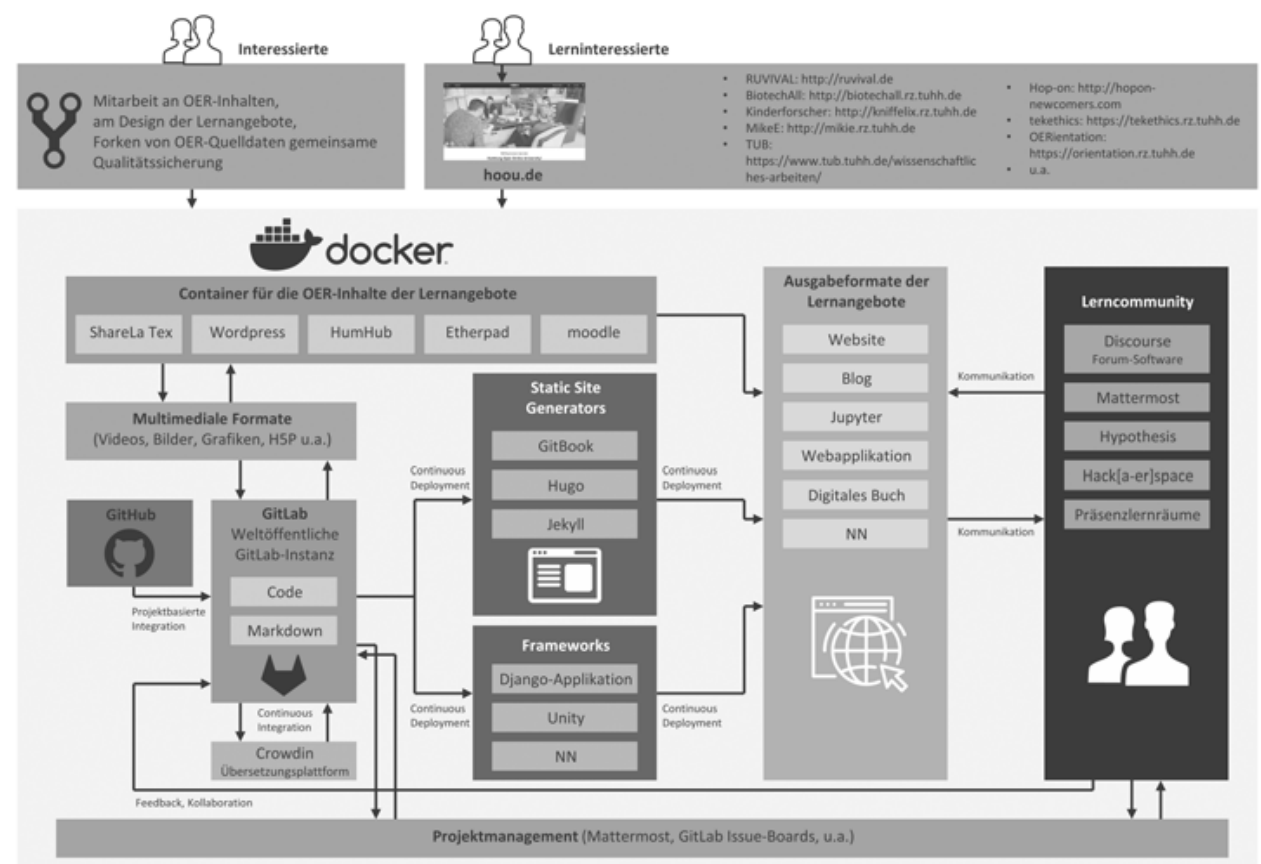

Das Experimentierfeld der HOOU an der TURH

Axel Durkop, Dr. Tina Ladwie. Prot. Or. Sonke Knutzen. Stand: 30.01 .2018 , vo.14

Abb. 3: Das technische Experimentierfeld der HOOU an der TUHH (eigene Darstellung).

Im Zuge der technischen Entwicklungen und Kooperationserfahrungen haben sich ebenso sozial orientierte Austauschformate an der TUHH entwickelt. $\mathrm{Zu}$ diesen zählt beispielsweise der Hackerspace ${ }^{13}$. Hier erprobt das Team der HOOU an der TUHH gemeinsam mit den HOOU-Projekten aus den verschiedenen Instituten an der TUHH, wie neue Konzepte und Ideen technisch umgesetzt werden können und die offene Lernangebote mittels verschiedener Open Source Tools

11 http://community.tuhh.de.

12 https://mikie.rz.tuhh.de/virtuellesLabor.html.

13 https://community.tuhh.de/c/hackerspace. 
unterstützt und realisiert werden können. Der Hackerspace findet wöchentlich statt und wird als offener Ort verstanden, an dem gemeinsam mit neuen technischen Möglichkeiten experimentiert werden kann, sich über didaktische Innovation ausgetauscht werden kann, aber auch Herausforderungen im Zuge der Entwicklung offener an die Zivilgesellschaft gerichtete Lehr- und Lernangebote platziert werden können.

Neben diesem Format gibt es die Arbeitsgruppe openTUHH. Seit 2017 widmet sich die AG der Aushandlung von Potenzialen der Digitalisierung für Studium und Lehre sowie Forschung und den Wegen zur Realisierung dieser Potenziale. Ziel der AG ist es, den digitalen Transformationsprozess an der TUHH aktiv aus strategischer, struktureller und kultureller Perspektive mitzugestalten. Da diese Aufgabe einen multiperspektivischen Blickwinkel erfordert, setzt sich die AG openTUHH aus dem Vizepräsidenten für Forschung und Lehre, dem Digitalisierungsbeauftragen für Lehre, dem Leiter und den Mitarbeitenden des Rechenzentrums, aus der Leiterin und den Mitarbeitenden der Bibliothek sowie dem HOOU-Team der TUHH zusammen.

Um für die Potenziale von Digitalisierung in der Lehre zu sensibilisieren und die Hochschulangehörigen auch bei der Realisierung zu unterstützen, wurden verschiedene Maßnahmen ergriffen. So ist 2018 aus der AG die openPolicy der TUHH entstanden. Darüber hinaus teilen die Mitglieder der AG regelmäßig ihr Wissen über INSIGHTS, einen Blog, der über das digitale Experimentierfeld der TUHH in Forschung und Lehre berichtet ${ }^{14}$. Flankierend dazu werden im Rahmen des wissenschaftlichen Arbeitens bei den Studierenden und durch die Graduiertenakademie Workshops zu digitalen Werkzeugen angeboten. Hierzu zählt unter anderem auch das Arbeiten mit GitLab, um Prinzipien der Kollaboration und der interdisziplinären Teamarbeit schon frühzeitig im Studium mithilfe von digitalen Werkzeugen zu vermittel $n^{15}$. Darüber hinaus bringt sich die AG im Sinne eines nationalen wie auch internationalen Wissensaustausches in andere Netzwerke ein und beteiligt sich an diversen Veranstaltungen, wie der Open Access Week oder Education Week.

Während die Zusammenführung der Open Source Tools auf technischer Ebene zur Erfüllung des Markenkerns der HOOU und der user-spezifischen Anforderungen beiträgt, ist die AG auf sozialer Ebene ein wichtiger Gelingensfaktor für die Realisierung offener, digital gestützter Lehr- und Lernprojekte im Rahmen der HOOU an der TUHH ${ }^{16}$.

14 https://insights.tuhh.de/de/.

15 http://collaborating.tuhh.de.

16 https://hoou.tuhh.de/projekte/. 


\section{Prototypische Projekte im Experimentierfeld der TU Hamburg}

Im Laufe der Vorprojekt- und Projektphase der HOOU sind zahlreiche Projekte im Experimentierfeld der HOOU an der TUHH konzeptionell entwickelt und realisiert worden. Das inhaltliche Spektrum umfasst Themen wie Artificial Intelligence, Migration, Klimawandel oder Armut. Neben der bestehenden Diversität der gewählten Themenschwerpunkte in den HOOU-Projekten, unterscheiden diese sich auch hinsichtlich der avisierten Zielgruppen. Tabelle 2 gibt einen Überblick über die aktuell laufenden HOOU-Projekte an der TUHH.

Tab. 2: Überblick zu aktuell laufenden HOOU-Projekten (Stand: 01.10.2019).

\begin{tabular}{|c|c|c|}
\hline Projekttitel & Zielgruppe & Link \\
\hline RUVIVAL & $\begin{array}{l}\text { Zielgruppen/Beteiligte: } \\
\text { Studierende der TUHH (zwei Kurse begleitend zum of- } \\
\text { fenen Lernangebot), } \\
\text { lokale und internationale Partnerinstitutionen der } \\
\text { TUHH, insbesondere Partner_innen in der Entwick- } \\
\text { lungsarbeit, } \\
\text { Fachpublikum, Politik und Verwaltung, NGOs in der in- } \\
\text { ternationalen Entwicklungsarbeit. }\end{array}$ & http://ruvival.de \\
\hline MikiE & $\begin{array}{l}\text { Zielgruppen/Beteiligte: } \\
\text { Studierende der Naturwissenschaften, Schülerinnen } \\
\text { und Schüler der Oberstufe sowie Personen, die an Bio- } \\
\text { technologie, Mikrobiologie und Nachhaltigkeit interes- } \\
\text { siert sind. }\end{array}$ & $\begin{array}{l}\text { https://mikie.rz. } \\
\text { tuhh.de }\end{array}$ \\
\hline Kniffelix & $\begin{array}{l}\text { Zielgruppen/Beteiligte: } \\
\text { Kinder und Jugendliche ab } 8 \text { Jahren, } \\
\text { Lehrer/innen und Eltern und angehende Pädagogen/ } \\
\text { innen, } \\
\text { Schüler-/innen der Abschlussklassen vor dem Studi- } \\
\text { um. }\end{array}$ & $\begin{array}{l}\text { https://kniffelix. } \\
\text { rz.tu-harburg.de }\end{array}$ \\
\hline RINOcloud & $\begin{array}{l}\text { Zielgruppen/Beteiligte: } \\
\text { Kinder und Jugendliche ab } 8 \text { Jahren, } \\
\text { Lehrer/innen und Eltern und angehende Pädagogen/ } \\
\text { innen. }\end{array}$ & $\begin{array}{l}\text { https://rinocloud. } \\
\text { hoou.tuhh.de }\end{array}$ \\
\hline tub.t\#OERials & $\begin{array}{l}\text { Zielgruppen/Beteiligte: } \\
\text { Studierende, Forschende und Lehrende insbesondere } \\
\text { der TUHH, die sich für offenes Publizieren, offene Bil- } \\
\text { dung und offene Wissenschaft interessieren. }\end{array}$ & $\begin{array}{l}\text { https://www.tub. } \\
\text { tuhh.de/tubtori- } \\
\text { als/ }\end{array}$ \\
\hline tekethics & $\begin{array}{l}\text { Zielgruppen/Beteiligte: } \\
\text { Studierende der HCU, der UHH sowie der TUHH sowie } \\
\text { die Zielgruppen der Bücherhallen. }\end{array}$ & $\begin{array}{l}\text { http://tekethics. } \\
\text { rz.tuhh.de }\end{array}$ \\
\hline
\end{tabular}




\section{Ausblick}

Basierend auf den eingangs erwähnten Rahmenbedingungen, ist auch bei der Entwicklung und Weiterentwicklung des Experimentierfeldes der HOOU an der TUHH eine zunehmende Komplexität zu beobachten, die nicht nur Kooperation im Hochschulverbund, sondern auch in der Hochschule erfordert. Durch die organisationalen Anforderungen an die Hochschule, wie beispielsweise datenschutzrechtliche, technische, aber auch personalwirtschaftliche Fragestellungen, ist die Zusammenarbeit mit unterschiedlichen Akteurinnen und Akteuren in den beschriebenen Handlungsfeldern unabdingbar. Die digitalen Strukturen eines solchen Experimentierfeldes sind sowohl auf technischer als auch sozialer Ebene zu gestalten und müssen nicht nur den originär bezogenen Leitideen der HOOU, sondern auch den individuellen Anforderungen der Nutzerinnen und Nutzer sowie der Kooperationspartnerinnen und -partner entsprechen. Die HOOU@TUHH muss als sozio-technisches Experimentierfeld ganzheitlich agieren, um die Entwicklung offener, digital gestützter Lehr- und Lernangebote unterstützen und voranbringen zu können.

\section{Literatur}

Dürkop, A.; Böttger, A.; Ladwig, T.; Knutzen, S. (2018): Ein technisches System für die kollaborative OER-Entwicklung im Experimentierfeld der TUHH. https://doi.org/10.15480/ 882.1653.

Dürkop, A.; Ladwig, T. (2016a): Der gemeinsame Weg zu einem Lernarrangement in der Hamburg Open Online University. Synergie. Fachmagazin für Digitalisierung in der Lehre 1, 76-77.

Dürkop, A.; Ladwig, T. (2016b): Neue Formen der Koproduktion von Wissen durch Lehrende und Lernende. https://doi.org/10.15480/882.1334.

Dürkop, A.; Ladwig, T. (2018): Das digitale Experimentierfeld für Lehre und Forschung. https:// insights.tuhh.de/de/das-digitale-experimentierfeld-fuer-lehre-und-forschung/.

Patton, J. (2014): User Story Mapping. Discover the whole story, build the right product. Sebastopol, CA: O'Reilly.

Schwalbe, C.; Peters, P.; Ladwig, T.; Jackewitz, I.; Göcks, M.: Knutzen, S. (2016): Innovationen und Entwicklungen in der HOOU. Grundprinzipien agiler Entwicklung im Hochschulkontext. Synergie. Fachmagazin für Digitalisierung in der Lehre 2, 42-43. 